\title{
Eficiência da avaliação pós-ocupação em conjuntos habitacionais
}

\author{
Efficiency of post-occupancy assessment in housing complexes \\ Eficiencia de la evaluación posterior a la ocupación en complejos habitacionales
}

\author{
Cynthia Rodrigues Silveira \\ ORCID: https://orcid.org/0000-0001-9798-869X \\ Pontifícia Universidade Católica de Goiás, Brasil \\ E-mail: cynthiaroodrigues@ hotmail.com \\ Lucia Maria Moraes \\ ORCID: https://orcid.org/0000-0002-9459-3948 \\ Pontifícia Universidade Católica de Goiás, Brasil \\ E-mail: Lucia.dhescmoradia@gmail.com
}

\begin{abstract}
Resumo
A pretensão incessante do governo em minimizar a escassez de residências inapropriadas no país, concebeu programas habitacionais brasileiros que empregam construções em massa e de baixo custo com o objetivo de amparar o maior número de famílias, em sua maioria sem acesso a uma moradia adequada. No entanto, a imposição de produzir o maior número de unidades residências com o capital disponível não constitui uma melhor condição de vida para as famílias alvo e consequentemente uma não diminuição do parâmetro habitacional nas cidades. A fim de compreender tais circunstâncias envoltas nas habitações de interesse social, pesquisas são empregadas na intenção de analisar conjunto habitacionais de modo a identificar adversidades e recomendar propostas que visam produzir melhoras significavas nas edificações, urbanização e inserção dos mesmos nas cidades. Diante disso, o presente trabalho buscou validar a eficiência da avaliação pós ocupação em conjuntos habitacionais através de uma revisão da literatura de natureza qualitativa, com base em estudos desenvolvidos pelo Instituto de Pesquisa Econômica Aplicada expondo questões positivas e negativas quanto a teoria e a aplicabilidade da análise, concluindo que a Analise de Pós Ocupação é eficiente em estudos voltados a empreendimento para a moradia social, devido ao seu parecer, o qual visa o ponto de vista do usuário e o desempenho da construção, atingindo resultados pertinentes.
\end{abstract}

Palavras-chave: APO; Déficit habitacional; Habitação de interesse social.

\begin{abstract}
The government's relentless attempt to minimize the scarcity of inappropriate homes in the country, designed Brazilian housing programs that employ mass and low-cost construction with the aim of supporting the largest number of families, most of whom do not have access to a complete home. However, an imposition to produce the largest number of residential units with available capital does not constitute a better living condition for the target families and, consequently, a non-reduction of the housing parameter in the cities. In order to understand such circumstances, involved in social housing, research is used with the intention of analyzing housing complexes in order to identify adversities and proposed proposals that aim to produce significant improvements in buildings, urbanization and their insertion in cities. In light of this, the present study sought to validate the efficiency of post-occupation assessment in housing estates through a literature review of a qualitative nature, based on studies developed by the Institute of Applied Economic Research exposing positive and negative questions regarding the theory and applicability of analysis, concluding that the Post-Occupancy Analysis is efficient in studies aimed at entrepreneurship for social housing, due to its opinion, which seen the user's point of view and the performance of the construction, reaching relevant results.
\end{abstract}

Keywords: APO; Housing deficit; Social interest housing.

\section{Resumen}

El incansable intento del gobierno de minimizar la escasez de viviendas inapropiadas en el país, diseñó programas de vivienda brasileños que emplean la construcción masiva y de bajo costo con el objetivo de apoyar al mayor número de familias, la mayoría de las cuales no tienen acceso a una vivienda completa. Sin embargo, la imposición de producir el mayor número de unidades residenciales con el capital disponible no constituye una mejor condición de vida para las familias objetivo y, en consecuencia, una no reducción del parámetro habitacional en las ciudades. Para comprender estas circunstancias, involucradas en la vivienda social, se utiliza la investigación con la intención de analizar desarrollos habitacionales con el fin de identificar adversidades y plantear propuestas que tengan como objetivo producir mejoras significativas en los edificios, la urbanización y su inserción en las ciudades. Ante esto, el presente estudio buscó validar la eficiencia de la valoración pos-ocupación en urbanizaciones a través de una revisión bibliográfica de carácter cualitativo, a partir de estudios desarrollados por el Instituto de Investigaciones Económicas Aplicadas, exponiendo 
interrogantes positivos y negativos sobre la teoría. y aplicabilidad del análisis, concluyendo que el Análisis PostOcupación es eficiente en estudios orientados al desarrollo de una vivienda social, debido a su opinión, que ve el punto de vista del usuario y el desempeño de la construcción, logrando resultados relevantes.

Palabras clave: APO; Déficit habitacional; Vivienda de interés social.

\section{Introdução}

O alto índice de pessoas desabrigadas, locadas em lugares de risco ou sem saneamento básico, ou moram de aluguel se caracteriza o déficit habitacional. Em outras palavras, é a falta de acesso da população a uma moradia digna, onde, a residência precisa dispor do acesso à água potável, energia, esgotamento sanitário, coleta de lixo, proximidade ao transporte público, lazer, cultura, educação, atendimento médico e segurança, requisitos básicos recomendado pela Organização das Nações Unidas (1948) e posteriormente reforçado pela Constituinte de 1988 e o Estatuto das Cidades em 2001.

O Brasil lida com o déficit habitacional desde a lei Áurea em 13 de maio de 1888, que aprovou a liberação dos escravos, estes vão para o meio urbano e posteriormente com a expansão industrial a partir da década de 1930. Estes fatos intensificaram o deslocamento populacional sem planejamento prévio, o qual acarretou a falta de moradia e o crescimento desordenado dos grandes centros urbanos, surgindo assim diversos assentamentos irregulares e moradias inadequadas, assim explicito por Nunes e Barreto Filho, (2020).

Diante disso, várias políticas públicas habitacionais foram desenvolvidas ao longo dos anos, como, o Instituto de Aposentadorias e Pensões (IAP), a Fundação Casa Popular (FCP), o Banco Nacional da Habitação (BNH) e o Programa Minha Casa Minha Vida (PMCMV), com o objetivo de promover à população de baixa renda, moradias adequadas como mostra Monteiro e Veras, (2017). No entanto, tais programas firmaram uma produção em massa de casas de baixo padrão, as quais dispõem o mínimo para uma edificação unifamiliar.

Segundo o Jornal Estadão (2020), em um estudo publicado pela Associação Brasileira de Incorporadores (Abrainc) mostra que o déficit habitacional no Brasil fechou 2019 em 7,797 milhões de moradias, isto demostra que mesmo com a produção em massa de casas de baixo padrão para atender o maior número de famílias possível, o número de desabrigados no país continuou crescendo de forma exorbitante. No entanto, dados da Fundação João Pinheiro (2019) registrou 5, 876 milhões de moradias, acredita que esse número vem crescendo devido ao grande número de despejos dos assentamentos urbanos e de famílias que moram de aluguel em função da Covid $19^{1}$.

Deste modo, gradativamente as residências direcionadas para a população sem moradia adequada, vem de certa forma diminuindo o padrão da construção tanto quanto a área construtiva, materiais de construção utilizado e a localização do conjunto habitacional, se fundamentando no discurso de construir o maior número de casas possíveis e consequentemente o valor exorbitante da terra urbanizada.

Contudo, o quesito reduzir o déficit habitacional deveria ser julgado de modo a proporcionar melhores condições de vida as famílias sem moradia apropriada, rejeitando a prática da produção em massa. Uma vez que, a edificação financiada pelo governo é significativamente pequena e insuficiente para uma família brasileira, onde a média de pessoas por residência segundo Camargo et al. (2013) é de 3,6 pessoas. No entanto, o mecanismo utilizado pelos programas habitacionais fomenta a desigualdade e segregação socioespacial, segundo Almeida, Reis \& Araújo (2020).

A avaliação pós ocupação (APO), consiste no parecer dos usuários em relação a um ambiente construído, determinando pontos positivos e negativos perante a utilização do espaço analisado. Esse sistema de apreciação das construções, começou a

\footnotetext{
${ }^{1}$ Covid19, é uma doença infeciosa causada pelo coronavírus da síndrome respiratória aguda grave. O vírus infetou pessoas no mundo todo, transformando-se em uma pandemia que resultou em instabilidade social e econômica global significativa.
} 
ser empregada no Brasil após a produção em massa de residências sociais, buscando qualificar o meio construído em associação a família que reside no local, a fim de demostrar possíveis falhas das edificações financiadas (Ono et al., 2018).

A APO vem se remodelando ao longo dos anos, mas, na atualidade ainda é muito empregada por pesquisadores que buscam avaliar as construções entregues pelo Estado em conjuntos habitacionais, com o objetivo de expor os problemas enfrentados pelos moradores após a apropriação do imóvel, a adoção dos resultados poderá proporcionar melhorias nas construções e moradias dignas para a famílias. A avaliação pós ocupação, pode ser utilizada para analisar ambientes diversos, no entanto, sua maior aplicação consiste em avaliar as condições técnicas construtivas e ambientais dos empreendimentos construídos para atender a população de menor poder aquisitivo.

\section{Referencial Teóricos}

A APO tem como princípio avaliar ambientes, independentemente de sua função, onde o importante é qualificar o desempenho construtivo e espacial, perante o ponto de vista de seus usuários. Desta forma, as ponderações da pós ocupação visa obter informações técnicas construtivas a fim de corrigir possíveis falhas e designar prováveis soluções, com o objetivo de conceber uma edificação satisfatória aos usuários, assim idealizado por Blachère (1966).

A intenção de avaliar o meio construído, foi proposto durante o Pós II Guerra ${ }^{2}$ pelo francês Gerard Blachère a mais de 50 anos. $\mathrm{O}$ autor acreditava que o ato de construir se dava para resolver problemas, no entanto, para realizar um projeto bem elaborado e que atenda aos requisitos de uso e satisfação era necessário saber construir, definido pelo Centre Scientifique et Technique du Bâtiment (2011).

Diante da diversidade de regras na construção Blachère fez campanha a favor das regras unificadas, contribuindo para o desenvolvimento das normas técnicas da International Organization for Standardization da (ISO) 6241, renomeada para ISO 19208 na atualidade, a qual tem como objetivo o desempenho de determinado objeto em relação ao seu uso (Centre Scientifique et Technique du Bâtiment, 2011).

No Brasil, a APO sucedeu durante a década de 1970, período da ditadura militar, quando foi criado o BNH e instituído o Sistema Financeiro de Habitação (SFH), o qual direcionava recursos para a produção em massa de residências sociais, com o objetivo de controlar o déficit habitacional no país (Medeiros, 2010). Essa promoção em larga escala de edificações realizadas pelo poder público, despertou curiosidade nos pesquisadores do Instituto de Pesquisas Tecnológicas do Estado de São Paulo (IPT) sobre a satisfação dos moradores em relação às edificações de interesse social. No entanto, a avaliação pós ocupação apenas se estabeleceu na década de 1980, especialmente nas instituições públicas de ensino superior (Ono et al., 2018). Deste modo, o processo de consolidação se deu por pesquisas direcionadas a habitações de interesse social.

Neste início do século XXI a APO ainda tem grande impacto quando se trata de conjuntos habitacionais, tendo como evidência a Plataforma da Coordenação de Aperfeiçoamento de Pessoal de Nível Superior (Capes), site do Ministério da Educação (MEC), que é responsável pela expansão e consolidação da pós-graduação, onde é possível ter acesso a produções científicas nacionais e internacionais (Capes, sd). Quando pesquisado por "avaliação pós ocupação" na plataforma, 4,4\% dos resultados são sobre pesquisas em shoppings, 4,4\% em museus, 7,45\% pesquisas de caráter ambiental, 10,3\% sobre referenciais da APO, 13,2\% pesquisas em ambientes escolares, 33,8\% sobre habitações sociais e 26,5\% sobre assuntos diversos. Ou seja, pesquisas voltadas para a aplicação da APO são em sua maioria ligada a conjuntos habitacionais, em especial os empreendimentos do PMCMV.

\footnotetext{
2 Após a Segunda Guerra Mundial (1939-1945) ocorreu uma expansão econômica, também conhecida como o boom econômico pósguerra ou Era de Ouro do capitalismo, um período de prosperidade econômica que durou até início de 1970, resultando em mudanças em diversas áreas de atividade.
} 
Na generalidade, a APO em conjuntos habitacionais tem como tarefa informar-se sobre a opinião dos moradores em relação ao uso da edificação. No entanto, Lay \& Reis (1994) reforça de que o foco não precisa ser somente a respeito do desempenho e a vivência na construção, mais a moradia como um todo, como, por exemplo, sua localização perante o território urbano, a disponibilidade de acervo ambiental, a qualidade do saneamento básico disposto, problemas construtivos entre outros tópicos.

A APO, busca dar orientações em todos os âmbitos de forma a propiciar melhorias ao conjunto habitacional, de modo a tornar o programa mais eficaz e satisfatório para a população que irá o auferir. Ou seja, as pesquisas não possuem o intuito de rebaixar os programas habitacionais, e sim de os aprimorar, pois, de 209,5 milhões de brasileiros 7,797 milhões não possuem moradia apropriada (Banco Mundial, 2020).

\section{Habitação de Interesse Social}

Em 1948 a Organização das Nações Unidas (ONU) declarou ser direito de todo cidadão ter acesso à moradia. Nessa situação, o Brasil dispôs de diversos programas habitacionais, como, por exemplo, o IAP e a FCP, e teve grande progresso na década de 70 com a primeira política pública instituída, em que o BNH era o órgão central como realça Miron (2008). Mas, somente em 1988 o assunto, habitação esteve presente na Constituição Federal, e passou a ser um direito constitucional no ano de 2000, quando a Emenda Constitucional n. 26 foi incorporada, incluindo o direito à moradia no país.

O déficit habitacional, como define Lima Neto, Furtado \& Krause (2013), é um parâmetro que aponta o número de pessoas sem moradia adequada, isto é, instalada em condições precárias, em coabitações forçadas, famílias que possuem pouca renda e pagam aluguel, ou ainda os domiciliados em imóveis de fins não residenciais. No geral, para Telli (2016) a falta de moradia quantifica a população de um país sem recursos para alcançar uma edificação própria. Á vista disso, foi desenvolvido as habitações de interesse social, uma solução governamental que busca promover a inclusão social de famílias de baixa renda a propriedade.

Deste modo, o governo disponibiliza meios de financiamentos conforme a classe social de cada família, isto é, o Plano Nacional da Habitação (PlanHab) ${ }^{3}$ dividiu o país em classes, a fim de facilitar o atendimento ao público-alvo de programas habitacionais como, o PMCMV, o qual tem como base o projeto instituído pelo BNH (Plano Nacional da Habitação, 2009).

Perante o exposto, tais programas habitacionais tinham ou tem como público-alvo a classe "E" e "D", que se refere a $32 \%$ das famílias brasileiras, as quais possuem uma renda mensal de até 3 salários-mínimos (sm). A classe "C", mais conhecida como classe média, corresponde a $52 \%$ da população brasileira, e possui uma faixa de 3 a $10 \mathrm{sm}$. Já as classes "A" e "B" correspondem a $16 \%$ da população e ficam de fora dos programas habitacionais por possuírem condições mais que suficientes para conquistar uma moradia adequada (Navarro \& Dias, 2009).

O financiamento disponibilizado pelo programa, conforme relato de Da Silva e Alves, (2014) exige uma entrada de $20 \%$ do valor do imóvel e um comprometimento de gasto mensal do mutuário, que não pode ultrapassar 30\% da renda familiar mensal bruta, por conta desses motivos os incentivos são em sua maioria, proporcionados as famílias da classe "C", por possuírem uma renda superior.

Por conta da proporção de famílias sem potencial para o financiamento do programa para adquirir imóveis, empreendimentos habitacionais começaram a ser construídos a fim de disponibilizar para as famílias de classe "E" e "D" residências com subsídio correspondido a quase 100\% do valor financiado (Cunha, 2020).

\footnotetext{
${ }^{3}$ O Plano Nacional de Habitação (PlanHab) planeja ações públicas e privadas, no objetivo de formular estratégias que permitam equacionar as necessidades habitacionais do país.
} 
Não obstante, comumente quando investigado a respeito do PMCMV em sites do Governo Federal, é possível encontrar separações por faixas, onde a FAIXA 1 se refere à classe "E" citada acima no texto, a FAIXA 1,5 a classe "D", e as FAIXAS 2 e 3 a classe "C". Deste modo, o público-alvo para aquisição de subsídios de moradia do PlanHab e do PMCMV são os mesmos, apenas possuem designações diferentes.

Contudo, as construções ofertadas para a população de baixa renda através dos conjuntos habitacionais não visam o conforto e sim atender o maior número de famílias, dado que, as edificações são construídas com menor custo possível para que o capital destinado produza o maior número de residências (Bonduki, 2017). No entanto, o fato de produzir quantidades maiores de moradias, não assegura condições de vida melhores para as famílias, tornando o programa de certo modo ineficiente e inadequado para a moradia digna.

Em outras palavras o programa propiciou de forma eficaz a reduzir o déficit habitacional, sem gerar melhores condições de moradia para a população de baixa renda, dado que as casas não seguem um perfil para cada família, são localizadas nas periferias da cidade, são insatisfatórias e os moradores realizam reformas após a ocupação e muitas das vezes não possuem todos os elementos do saneamento básico, análise feita por Lima et al., (2010) o que deverá ser pesquisado e analisado em um estudo de caso em outro momento.

Todos os programas habitacionais desenvolvidos ao longo dos anos no Brasil foram de suma importância para a inclusão das famílias de baixa renda a propriedade, contudo, melhorias poderiam ser realizadas a fim de tornar o programa mais eficiente e satisfatório para seus usuários.

\section{Metodologia}

Os procedimentos metodológicos adotados neste trabalho fundamentaram-se na contextualização teórica da eficiência da APO. Os dados analisados para o presente artigo se deram a partir da leitura de livros, dissertações, teses e artigos de congressos nacionais e internacionais, assim como consultas em instituições públicas e na Internet, o que salienta ser uma pesquisa qualitativa baseada em estudos teóricos.

O Instituto de Pesquisa Econômica Aplicada (Ipeia, p. 9, 2016), "entende-se que a aplicação da APO deveria estar implícita no cotidiano de todos os profissionais nos campos da arquitetura, do urbanismo, da engenharia civil e do design, com o intuito de ampliar a qualidade de seus processos de projetos e produtos". A importância do "saber como se comporta efetivamente o usuário no ambiente construído deveria ser uma prática corrente entre os diversos agentes deste mercado em diferentes esferas (pública, institucional, privada, coorporativa, entre outras)". No entanto, é preliminar essas análises nas rotinas do mercado imobiliário e nos órgãos do governo no Brasil.

A aquisição de arquivos desse trabalho decorreu da busca em plataformas acadêmicas pelas palavras chaves, "avaliação pós ocupacional", "post-occupancy evaluation AND social housing" e "habitações de interesse social".

Em geral, o estudo de caso é exploratório e descritivo, o qual coloca em questão fenômenos reais em debate, indagando o "como" e "por que" de certos eventos (Yin, 2015). Deste modo, o artigo apresenta um estudo exploratório qualitativo acerca da APO em conjuntos habitacionais, expondo os pontos positivos e negativos diante da metodologia utilizada quantificando se a mesma é eficiente na apreciação de dados e sugestões.

\section{Resultados}

É fato que o déficit habitacional no país é alarmante, e as moradias designadas para contê-lo são ínfimas, localizadas nas periferias da cidade, as construções são de baixa qualidade e muitas das vezes não possuem todos os equipamentos de saneamento básico, e isso se deve ao fato do governo economizar o máximo possível na produção para que um maior número de 
famílias possa ser atendido (Lima et al., 2010) e que a especulação da terra passou a ser um fator preponderante no território urbano.

No entanto, como dito anteriormente, atender ao maior número de pessoas com o objetivo de conter o déficit, não significa proporcionar melhores condições de vida aos usuários dos programas habitacionais. Em países desenvolvidos, como, por exemplo, Suíça, Noruega, Alemanha e outros, tem se incluído, características como tamanho e composição da família nas residências sociais, isto é, as edificações financiadas pelo governo variam segundo a necessidade de cada grupo, e isso as tornam mais eficientes que os programas habitacionais brasileiros (Ono et al., 2018).

Para que os programas habitacionais brasileiros se tornem mais eficientes é necessário promover certas mudanças, as quais considerem a opinião do público-alvo do programa (Pinheiro \& Gunther, 2008). Deste modo, o Brasil não estará apenas se preocupando com os números de um parâmetro e sim com os resultados satisfatórios da população foco.

A APO seria o procedimento avaliativo mais indicado para detectar mudanças significativas do ponto de vista do usuário, a fim de tornar os programas e os empreendimentos mais satisfatórios. Visto que, as sugestões partem do ponto de vista do morador, para que o mesmo possua um ambiente mais confortável e adequado para sua vivência familiar.

Segundo Villa e Ornstein (2016), a APO é um conjunto de procedimentos que visam verificar o atendimento as necessidades objetivas e subjetivas do usuário em relação ao espaço construído, certificando assim, a eficiência em solucionar questões habitacionais através de um ponto de vista pessoal.

No entanto, para atingir resultados significativos é necessário compreender sobre a APO e avaliar o maior número possível de residentes. Para isso, pode-se contar com pesquisadores internacionais, como, por exemplo, Preiser, Rabinowitz e White que publicaram em 1988 o manual da avaliação pós ocupacional "Post-Occupancy Evaluation", e Marcos e Sarkissian que lançaram em 1986 o livro "Housing As If People Mattered". Equivalente a tais bibliografias, o Brasil dispõe de pesquisadores como, por exemplo, Ornstein e Roméro que publicaram em 1992 "Avaliação Pós-Ocupação do Ambiente Construído”, Pinheiro e Gunther com "Métodos e Pesquisas nos Estudos Pessoa-Ambiente", entre outros.

Além dos registros de pesquisadores, a avaliação pós-ocupação compara a edificação com as normas, como a, ISO 19208, a NBR 15575 e outras, no intuito de observar o desempenho do ambiente construído, e concluir se está adequado para o uso. Ou seja, além de considerar a opinião e grau de satisfação do usuário, ela avalia a performance da construção, buscando aprimorar o espaço estudado em duas perspectivas diferentes, a teoria que são as normas, e a prática que é o grau de satisfação dos residentes quanto a edificação (Blachère, 1966).

$\mathrm{O}$ fato de a APO julgar duas perspectivas diferentes de modo a proporcionar melhores resultados a ambiência de uma construção, a qualifica para ponderar reparos em conjuntos habitacionais, pois além de proporcionar conforto aos usuários, pode favorecer um melhor desempenho a edificação (Preiser, White \& Rabinowitz, 2015).

No entanto, a limitante da APO em pesquisas de habitações sociais é a produção em massa, isto é, o número de casas em um único conjunto habitacional pode chegar a 5.500 unidades que é o caso do Complexo Aluízio Campos o maior do país (Governo Federal, 2018). Por ser uma avaliação de caráter pessoal, é necessário que o maior número de pessoas seja entrevistado, a fim de obter dados significativos com a menor porcentagem de erro.

A APO tem sido empregada como um processo final, sendo utilizada para obtenção de informações posteriormente a construção do ambiente. Porém, deve ser empregada a partir do início de uma obra, a fim de coletar dados que englobem todo o ciclo de vida da habitação, de modo a torná-la mais agradável e com um bom funcionamento, evitando possíveis descontentamentos após a ocupação do ambiente construído (Ono et al., 2018). 


\section{Conclusão}

A habitação possui extrema importância para a vida do ser humano desde os corpos sociais mais remotos da história, servindo de abrigo, proteção contra intempéries e quaisquer outros riscos a que o indivíduo possa estar exposto. No entanto, os anos se passaram e às moradias tomaram um novo foco, satisfazer seus usuários, tornando as residências um local de descanso, lazer e convívio familiar, de modo que corresponda com os parâmetros de cada família, isto é, esteja apta ao número de pessoas residentes no local, prazeres individuais, conforto térmico, acústico e vários outros desempenhos que a construção pode desenvolver para o bem-estar de uma família.

A comodidade de um ambiente familiar não deve estar ligada à sua condição financeira, visto que tanto a ONU quanto pela Constituição Brasileira é direito de todo cidadão ter acesso a uma moradia apropriada. De certa forma os programas habitacionais destinados a promover melhores condições de moradia à essas famílias estão ineficientes, quanto a localização dos bairros, a qualidade dos materiais implantados nas obras, a dimensão das edificações que em quase $100 \%$ dos casos passam por reformas, a carência de alguns meios de saneamento básico, segurança, pois muitas das vezes os conjuntos habitacionais passam a ser inseguros, falta de acervo ambiental, desigualdade social, entre outros impasses são enfrentados pelos moradores desses bairros periféricos.

Sendo assim, melhorias precisam ser realizadas de modo a oferecer a população de baixa renda, moradias mais satisfatórias e similares ao perfil dos usuários. E diante da presente pesquisa é possível contemplar a eficiência da APO em conjuntos habitacionais tanto pela abundância de informações e dados a serem obtidos, quanto pela fundamentação da avaliação, que se baseia na satisfação do proprietário em relação ao ambiente construído, podendo ser ele por ambiência ou desempenho da edificação.

Não é possível, engenheiro ou arquiteto urbanista projetar uma edificação satisfatória sem conhecer o perfil do usuário, diante disso, mais uma vez mostra se a ineficiência das habitações sociais. Contudo, os programas habitacionais seriam facilmente capazes de criar um ambiente pós ocupação sem controversas se fossem devidamente planejados usufruindo da APO desde o princípio da criação do programa pelos governos municipais, estaduais e federal.

O fato é que a avaliação pós-ocupacional traz progressos tanto para projetos em fase inicial, quanto para projetos finalizados. Deste modo, a APO pode trazer melhorias para os futuros conjuntos habitacionais tendo como base os concluídos, assim como, é capaz de aperfeiçoar os finalizados, implantando os elementos inexistentes de saneamento básico, incorporado acervo ambiental, disponibilizando plantas de flexibilidade espacial a fim de auxiliar nas reformas pós ocupação, dentre outros métodos.

A APO busca métodos de implantar um ambiente qualificado para seus usuários, de modo a proporcionar conforto, satisfação e segurança. Uma boa ambiência para um convívio familiar não deve ser de desfrute apenas de famílias com boas condições financeiras e sim para toda a população.

Á vista da importância em gerar ambientes agradáveis à população de baixa renda, recomenda-se para trabalhos futuros, uma aplicação da APO em conjuntos habitacionais já ofertados, analisando as principais reformas realizadas pelos moradores pós ocupação, no intuito de obter informações sobre alterações quanto a adversidades e modificações para melhorar a convivência na edificação. Desta forma, se obteria dados suficientes para aperfeiçoar habitações de interesse social futuras, assim como, proporcionaria possíveis soluções para o bairro foco da pesquisa.

\section{Referências}

Almeida, R. M., Reis, R. B., \& de Araújo, P. S. R. (2020). Saneamento e Saúde Ambiental no entorno de áreas com habitação social em Salvador-BA. Research, Society and Development, 9(7).

Banco Mundial. (2020). Indicadores de Desenvolvimento Mundial. 2021. https://www.google.com.br/publicdata/explore?ds=d5bncppjof8f9_. 
Blachère, G. (1966). Savoir bâtir: habitabilité, durabilité, économie des bâtiments. Eyrolles.

Bonduki, N. (2017). Origens da habitação social no Brasil: arquitetura moderna, lei do inquilinato e difusão da casa própria. São Paulo: Estação Liberdade, $7^{\mathrm{a}}$ ed.

Camargo, C. F., Curralero, C. R. B., Licio, E. C., \& Mostafa, J. (2013). Perfil socioeconómico dos beneficiários do programa Bolsa Família. IPEA. Programa Bolsa Família: uma década de inclusão e cidadania. Brasília.

Coordenação de Aperfeiçoamento de Pessoal de Nível Superior. CAPES. (Sd.). Conheça a Plataforma Capes de Educação Básica. 2021. https://eb.capes.gov.br/sobre-plataforma.

Centre Scientifique et Technique du Bâtiment. CSTB. (2011). Gérard Blachère, scientifique du bâtiment. 2021. http://www.cstb.fr/archives/webzines/editions/webzine-du-4-juillet-2011/gerard-blachere-scientifique-du-batiment.html.

Cunha, G. A. C. (2020). Déficit Habitacional: O tamanho da desigualdade social no Brasil. Boletim Economia Empírica.

Da Silva, C. F. D., \& Alves, T. W. (2014). Dinâmica dos financiamentos habitacionais nos municípios do Rio Grande do Sul de 2006 a $2010:$ uma avaliação do Programa" Minha Casa, Minha Vida". Revista de Administração Pública, 48(1), 27-54.

Fundação João Pinheiro. (2019). Déficit habitacional no Brasil. Belo Horizonte. Centro de Estatísticas e Informação.

Governo Federal. (2018). Ministério do Desenvolvimento Regional. 2021. https://www.gov.br/mdr/pt-br/noticias/ministro-visita-maior-conjunto-habitacionaldo-minha-casa-minha-vida.

Jornal Estadão. (2019). Déficit habitacional é recorde no país. Economia. 2021. https://economia.estadao.com.br/noticias/geral,deficit-habitacional-e-recordeno-pais, 70002669433 .

Lay, M. C. D., \& Reis, A. T. D. L. (1994). The impact of housing quality on the urban image. The Urban Experience. London: Chapman and Hall, 85(98), 74.

Lima, F. R., Cosenza, C. A. N., Maria, A., \& Rossi, G. (2010). Localização de Empreendimentos Habitacionais, uma Abordagem Metodológica. Canela. ENTAC. Lima Neto, V. C., Furtado, B. A., \& Krause, C. H. (2013). Estimativas do déficit habitacional brasileiro (PNAD 2007-2012). IPEA. São Paulo.

Medeiros, S. R. F. Q. (2010). BNH: outras perspectivas. Natal.

Miron, L. I. G. (2008). Gerenciamento dos requisitos dos clientes de empreendimentos habitacionais de interesse social: proposta para o Programa Integrado Entrada da Cidade em Porto Alegre/RS. Rio Grande do Sul.

Monteiro, A. R., \& Veras, A. T. D. R. (2017). A questão habitacional no Brasil. Mercator (Fortaleza), 16.

Navarro, M., \& Dias, E. C. (2009). O financiamento habitacional da “nova classe média”. Conjuntura da Construção, 7(2), 10-11.

Nunes, I. S., \& Barreto Filho, B. F. (2020). Programa Minha Casa Minha Vida (PMCMV). Pensar Geografia, 4(1), $32-54$.

Ono, R., Ornstein, S. W., Villa, S. B., \& França, A. J. G. L. (2018). Avaliação pós-ocupação: na arquitetura, no urbanismo e no design. Oficina de Textos.

Organização das Nações Unidas. ONU. (1948). Declaração Universal dos Direitos Humanos. 2020. http://www.dudh.org.br/wpcontent/uploads/2014/12/dudh.pdf

Pinheiro, J. Q., \& Günther, H. (2008). Métodos de pesquisa nos estudos pessoa-ambiente. Casa do Psicólogo.

Plano Nacional da Habitação. (2019). Plano Nacional de Habitação. Versão para debates Brasília: Ministério das Cidades/ Secretaria Nacional de Habitação Primeira impressão, 212 p.

Preiser, W. F. E, White, E., \& Rabinowhitz, H. (2015). Avaliação pós-ocupação (Routledge Revivals). Routledg.

Telli, F. H., \& Librelotto, L. I. (2016). Sistemas Construtivos Aplicados à HIS-etapa 2. Florianópolis. P.20.

Villa, S. B., \& Ornstein, S. W. (2016). Qualidade ambiental na habitação-avaliação pós-ocupação. Oficina de Textos.

Villa, S. B., Saramago, R. D. C. P., \& Garcia, L. C. (2016). Desenvolvimento de metodologia de avaliação pós-ocupação do programa minha casa minha vida: Aspectos funcionais, comportamentais e ambientais. Ipea. Brasília.

Yin, R. K. (2015). Estudo de Caso: Planejamento e métodos. Bookman editora. 